\title{
Temperature Dependence of Material Behaviour at High Strain-Rate
}

\author{
M. Scapin ${ }^{1} \cdot$ P. Verleysen ${ }^{2} \cdot$ M. Hokka ${ }^{3} \cdot$ N. Bahlouli ${ }^{4}$
}

Published online: 31 July 2019

(c) Society for Experimental Mechanics, Inc 2019

In recent years, interest in material characterization at high strain-rates while varying the temperature has been continuously increasing. Consequently, the study and modelling of material behavior in such conditions has been promoted. In many applications such as machining, metal forming, high velocity impact or high energy deposition of metals, materials are deformed at very high rates. This produces self-heating to high temperatures due to adiabatic processes. In this case, the stress-strain response will be a balance between the effects of hardening (due to strain and strain-rate) and thermal softening. In other cases, the working temperature may be different to room temperature. Hence both the mechanical response of the material and the effect of strain-rate should be investigated in the domain of interest. At high temperature, materials generally become much more ductile and can also exhibit microstructural changes due to recrystallization phenomena. By contrast, at low temperatures the material strength usually increases and the mechanical behavior changes from ductile to brittle.

From these considerations, it appears evident that temperature and strain-rate are variables of fundamental importance in the prediction of the mechanical response of materials, playing an important role in many deformation processes. Hence, it is clear there is a need to define proper material models which could be implemented in numerical Finite Element simulations from which it should be possible to predict and estimate the responses of structures, components and materials under different loading conditions and

M. Scapin

martina.scapin@polito.it

1 Department of Mechanical and Aerospace Engineering, (DIMEAS), Politecnico Di Torino, Corso Duca degli Abruzzi 24, 10129 Torino, Italy

2 Department of Materials Science and Engineering, Ghent University, Ghent, Belgium

3 Engineering Materials Science, Tampere University, FI-33101 Tampere, Finland

4 ICube, Université de Strasbourg/CNRS, Strasbourg, France scenarios. Of course, the development of methodologies and facilities for the complete investigation of the mechanical response of materials in the whole temperature and strainrate field of interest is required and has to be addressed, by also taking into account the fact that temperature and strain-rate are mutually related. This means that the thermal effects obtained from quasi-static tests cannot always be used to predict material response under dynamic loading conditions. Moreover, this reveals that in order to consider the coupled effects of temperature and strain-rate, material models should be used in which the thermal component of stress is also considered.

It is with these considerations and goals that this special issue of the Journal of Dynamic Behavior of Materials has been assembled with the specific focus on the Temperature Dependence of Material Behaviour at High Strain-rate. The special issue covers several aspects of temperature effects on the dynamic behaviour of materials from the broad international community, with the aim to collect quality research findings and discussion on metals, composites, polymers, glass, sand, soil, and ice involving both experimental and modelling methodologies.

The guest editors gratefully acknowledge the technical contributions of all the authors and thank Dr. Eric N. Brown, Editor-in-Chief of Journal of Dynamic Behavior of Materials and the entire DYMAT Association (European association for the promotion of research into the dynamic behaviour of materials and its applications) for making the issue possible.

Publisher's Note Springer Nature remains neutral with regard to jurisdictional claims in published maps and institutional affiliations. 